\title{
Suicide Attempt Following Pacemaker Implantation in an Eighty-Three-Year-Old Male: A Case Report
}

\author{
Pedro Pallangyo a, b, e, Lucy Mgopa ${ }^{\mathrm{c}}$, Jalack Millinga ${ }^{\mathrm{d}}$, Smita Bhalia ${ }^{\mathrm{b}}$, Naairah R. Hemed ${ }^{\mathrm{a}}$, \\ Zabella Mkojera $^{\text {a }}$, Happiness J. Swai ${ }^{a}$, Polycarp Seraphine ${ }^{b}$, Rydiness Mulashanid, \\ Baraka Ndelwa $^{\mathrm{b}}$, Tulizo Shemu ${ }^{\mathrm{b}}$, Mohamed Janabi ${ }^{\mathrm{b}}$
}

\begin{abstract}
As the global population ages, cardiac pacing procedures are rising exponentially to keep pace with the increasing incidence of bradyarrhythmias. The efficacy of pacemakers is well established, but recipients may have poor psychosocial adaptation leading to development or exacerbation of mental disorders, that may manifest with anxiety, depressive symptoms or rarely suicidal tendencies. An 83-year-old male of African descent was referred to us for evaluation and expert management. He came with chief complaints of general body malaise, light-headedness, chest pain and fainting spells for about 6 months. He was diagnosed with hypertension 4 years prior, and there was no history of mental illness in the patient or his family. Echocardiography $(\mathrm{ECHO})$ revealed features of hypertensive heart disease while electrocardiogram (ECG) showed features of third-degree heart block. He underwent successful pacing with a resultant ventricular paced rhythm. The patient was stable and symptom free post pacing, but on the fourth day he jumped off the window of the ward in the hospital's second floor. Post suicide attempt examination revealed epistaxis, right periorbital hematoma with a temporal lacerated wound and deformed ankles bilaterally. ECG showed a ventricular paced rhythm and the chest radiograph showed an intact pacemaker. Ophthalmological review was evident for right sided blepharospasm with massive chemosis and bilateral constricted reactive pupils. Radiological investigations showed right orbital fracture, stable C5 and C6 fractures, and bilateral bimalleolar fractures with ankle dislocation. Neurosurgical review was unremarkable and psychiatric review could not be performed. The patient died $18 \mathrm{~h}$ after the suicide attempt incidence. Emotional disturbances post pacing impairs the quality of life and in the worst case scenario could lead to unanticipated ces-
\end{abstract}

Manuscript submitted October 8, 2019, accepted November 29, 2019

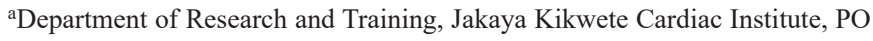
Box 65141, Dar es Salaam, Tanzania

${ }^{b}$ Department of Adult Cardiology, Jakaya Kikwete Cardiac Institute, PO Box 65141, Dar es Salaam, Tanzania

${ }^{\mathrm{c}}$ Department of Psychiatry and Mental Health, Muhimbili University of Health and Allied Sciences, PO Box 65001, Dar es Salaam, Tanzania

dDepartment of Nursing, Jakaya Kikwete Cardiac Institute, PO Box 65141, Dar es Salaam, Tanzania

${ }^{\mathrm{e}}$ Corresponding Author: Pedro Pallangyo, Jakaya Kikwete Cardiac Institute, PO Box 65141, Dar es Salaam, Tanzania. Email: pedro.pallangyo@gmail.com

doi: https://doi.org/10.14740/jmc3383 sation of life. In view of this, thorough evaluation and monitoring of the patient's psychological well-being both pre and post pacing is paramount.

Keywords: Pacemaker; Suicide; Psychological adaptation; Conduction disorders; Symptomatic bradycardia; Complete heart block; Third- degree heart block

\section{Introduction}

Cardiac rhythm disorders comprise an important epidemiological and public health problem worldwide. Arrhythmias are associated with increased morbidity and mortality, poor health-related quality of life and high cost of healthcare [1]. As the global population ages, the incidences of arrhythmias and its associated consequences are exhibiting a dramatic upsurge [2-4]. Consequently, cardiac pacing procedures are rising exponentially to keep pace with the increasing incidence of bradyarrhythmias [5-9].

In spite of the well-established potential in improving the quality of life and increase survival prospects [10-13], psychological adaption after pacing can be challenging to patients regardless of their baseline mental status [14-17]. For instance, rates of anxiety have ranged between $13.1-81.8 \%$ among pacemaker patients $[14,16,18]$. Similar pattern is observed in clinical depression with reported rates between $5.2 \%$ and $45.5 \%$ post pacing $[14,16,18]$. On the other hand, pacemaker associated suicide is relatively infrequent and the reported cases so far have featured patients with an underlying psychiatric illness $[19,20]$. In this report we present a case of suicide attempt following pacing in an 83-year-old man of African descent.

\section{Case Report}

An 83-year-old male of African descent was referred to us from Tanzania's Southern Highlands for investigations and expert management. He came with chief complaints of general body malaise, light-headedness, chest pain and fainting spells for about 6 months. His past medical history was remarkable for prostatectomy for benign prostatic hyperplasia (BPH) about 9 years ago. He was diagnosed with hypertension 4 years prior 
and his current medications included telmisartan $40 \mathrm{mg}$ once daily (od), atorvastatin $20 \mathrm{mg}$ od, and furosemide $40 \mathrm{mg}$ od. Neither the patient nor his family had reported previous history of mental illness.

On examination, he was oriented, well-kempt with an ageappropriate appearance. His body mass index (BMI) was 20.0 $\mathrm{kg} / \mathrm{m}^{2}$ (weight $51.1 \mathrm{~kg}$ and height $1.6 \mathrm{~m}$ ) and had stable vitals (blood pressure (BP) 131/90 mm Hg, respiratory rate (RR) 22 breaths/min, temperature $36.8{ }^{\circ} \mathrm{C}$, and oxygen saturation $99 \%$ in room air) except for bradycardia (pulse rate (PR) 31). Hematological and biochemical tests were evident for an iron deficiency anemia (hemoglobin (Hb) $9.5 \mathrm{~g} / \mathrm{dL}$, mean corpuscular volume (MCV) $78.9 \mathrm{fL}$, mean corpuscular hemoglobin (MCH) $23.7 \mathrm{pg} / \mathrm{cell}$, and red cell distribution width (RDW) $18.1 \mathrm{fL}$ ) otherwise normal. Serological tests for human immunodeficiency virus (HIV), syphilis, and hepatitis B and C were negative. Echocardiography (ECHO) revealed mild left ventricular hypertrophy (LVH) with preserved systolic functions (ejection fraction (EF) 53\%). Electrocardiogram (ECG) showed features of a third-degree heart block with a rate of 31 . He underwent pacing and Medtronic SENSIA SESR01 SNNWM46678 was implanted successfully with a resultant ventricular paced rhythm. Mental status evaluation of the patient before and after pacing was not performed.

The patient was stable and symptom free post pacing, but on the fourth day he jumped off the window of the ward in the hospital's second floor where he was admitted. He was then rushed to the emergency department. Post suicide attempt examination revealed epistaxis, right periorbital hematoma with a temporal lacerated wound and deformed ankles bilaterally. ECG showed a ventricular paced rhythm and the chest radiograph showed an intact pacemaker. Ophthalmological review was evident for a right sided blepharospasm with massive chemosis and bilateral constricted reactive pupils. Radiological investigations showed right orbital fracture, stable C5 and C6 fractures, and bilateral bimalleolar fractures with ankle dislocation. Neurosurgical review was unremarkable and psychiatric review could not be performed. The patient died $18 \mathrm{~h}$ after the suicide attempt incidence. The patient did not undergo autopsy due to refusal from the next of keen for cultural reasons.

Ethical clearance was sought from the Directorate of Research of Jakaya Kikwete Cardiac Institute.

\section{Discussion}

Pacemakers are highly effective electrotherapeutic devices of vital importance to patients with abnormalities of the atrioventricular (AV) conduction system. Since its debut in 1958, pacemakers have undergone remarkable transformation in terms of size, battery longevity and remote monitoring ability amongst others [21]. The efficacy of pacemakers is well established, but recipients may have poor psychosocial adaptation leading to development or exacerbation of mental disorders, that may manifest with anxiety, depressive symptoms or rarely suicidal tendencies [14-20].

Emotional disturbances among pacemaker patients have been understudied despite increasing number of pacing pro- cedures globally. Psychological evaluation of patient pre and post pacing is essential to pick the undetected coexisting psychiatric disorders and mood disorders ensuing from poor psychological adaptation post implantation respectively. Early recognition of such is fundamental to providing timely intervention with resultant better clinical outcomes and improved health-related quality of life post pacing.

In the case presented, neither pre- nor post-pacing mental status evaluation was performed. Although the intended lifesaving intervention was successful, the patient died of an unprecedented cause. Arguably, if the attending clinicians did a post-procedure mental status evaluation then mental instability should have been picked up and intervened timely. This case report illustrates the extreme consequences of poor psychological adaptation to a permanent pacemaker, and it underscores the importance of continued psychological evaluation to such patients.

\section{Conclusions}

Despite of its rarity, emotional disturbances post pacing impairs the quality of life and in the worst case scenario could lead to premature cessation of life. In view of this, thorough evaluation and monitoring of patient's psychological well-being both pre and post pacing is paramount.

\section{Acknowledgments}

The authors are grateful to the staff of the Jakaya Kikwete Cardiac Institute for their hard work in daily patient care.

\section{Financial Disclosure}

None to declare.

\section{Conflict of Interest}

The authors declare that they have no competing interests.

\section{Informed Consent}

Written informed consent was obtained from the patient's next of kin for publication of this case report.

\section{Author Contributions}

PP, LM and JM took the history and performed the physical examination. PS and TS implanted the pacemaker. NRH, HJS, $\mathrm{ZM}, \mathrm{SB}, \mathrm{BN}$ and $\mathrm{MJ}$ participated in clinical management of the patient during hospitalization. PP wrote the initial draft of the manuscript. All authors reviewed and contributed to the final version of this case report. 


\section{Abbreviations}

AV: atrioventricular; BMI: body mass index; BP: blood pressure; BPH: benign prostatic hyperplasia; ECG: electrocardiogram; ECHO: echocardiography; $\mathrm{EF}$ : ejection fraction; $\mathrm{Hb}$ : hemoglobin; HIV: human immunodeficiency virus; $\mathrm{MCH}$ : mean corpuscular hemoglobin; MCV: mean cell volume; PR: pulse rate; RR: respiratory rate; RDW: red blood cell distribution width

\section{References}

1. Murakoshi N, Aonuma K. Epidemiology of arrhythmias and sudden cardiac death in Asia. Circ J. 2013;77(10):2419-2431.

2. Rudy Y. Noninvasive imaging of cardiac electrophysiology and arrhythmia. Ann N Y Acad Sci. 2010;1188:214221.

3. Chow GV, Marine JE, Fleg JL. Epidemiology of arrhythmias and conduction disorders in older adults. Clin Geriatr Med. 2012;28(4):539-553.

4. Bradshaw PJ, Stobie P, Knuiman MW, Briffa TG, Hobbs MS. Trends in the incidence and prevalence of cardiac pacemaker insertions in an ageing population. Open Heart. 2014;1(1):e000177.

5. Mond HG, Irwin M, Ector H, Proclemer A. The world survey of cardiac pacing and cardioverter-defibrillators: calendar year 2005 an International Cardiac Pacing and Electrophysiology Society (ICPES) project. Pacing Clin Electrophysiol. 2008;31(9):1202-1212.

6. Mond HG, Proclemer A. The 11th world survey of cardiac pacing and implantable cardioverter-defibrillators: calendar year 2009-a World Society of Arrhythmia's project. Pacing Clin Electrophysiol. 2011;34(8):1013-1027.

7. Ector H, Vardas P. Current use of pacemakers, implantable cardioverter defibrillators, and resynchronization devices: data from the registry of the European Heart Rhythm Association. Eur Heart J Suppl. 2007;9(Suppl 1):144-149.

8. Bradshaw PJ, Stobie P, Briffa T, Hobbs MS. Use and longterm outcomes of implantable cardioverter-defibrillators, 1990 to 2009. Am Heart J. 2013;165(5):816-822.

9. Vellone E, Rega ML, Galletti C, et al. Anxiety and depression before and after a pacemaker implantation: a comparative Study. Int Nurs Persp. 2008;8(3):93-99.

10. Oto MA, Muderrisoglu H, Ozin MB, Korkmaz ME, Karamehmetoglu A, Oram A, Oram E, et al. Quality of life in patients with rate responsive pacemakers: a randomized, cross-over study. Pacing Clin Electrophysiol. 1991;14(5 Pt 1):800-806.

11. Linde-Edelstam C, Nordlander R, Unden AL, OrthGomer K, Ryden L. Quality-of-life in patients treated with atrioventricular synchronous pacing compared to rate modulated ventricular pacing: a long-term, double-blind, crossover study. Pacing Clin Electrophysiol. 1992;15(10 Pt 1):1467-1476.

12. Lukl J, Doupal V, Heinc P. Quality-of-life during DDD and dual sensor VVIR pacing. Pacing Clin Electrophysiol. 1994;17(11 Pt 2):1844-1848.

13. Lamas GA, Orav EJ, Stambler BS, Ellenbogen KA, Sgarbossa EB, Huang SK, Marinchak RA, et al. Quality of life and clinical outcomes in elderly patients treated with ventricular pacing as compared with dual-chamber pacing. Pacemaker Selection in the Elderly Investigators. N Engl J Med. 1998;338(16):1097-1104.

14. Newall EG, Lever NA, Prasad S, Hornabrook C, Larsen PD. Psychological implications of ICD implantation in a New Zealand population. Europace. 2007;9(1):20-24.

15. Leosdottir M, Sigurdsson E, Reimarsdottir G, Gottskalksson G, Torfason B, Vigfusdottir M, Eggertsson S, et al. Health-related quality of life of patients with implantable cardioverter defibrillators compared with that of pacemaker recipients. Europace. 2006;8(3):168-174.

16. Duru F, Buchi S, Klaghofer R, Mattmann H, Sensky T, Buddeberg C, Candinas R. How different from pacemaker patients are recipients of implantable cardioverter-defibrillators with respect to psychosocial adaptation, affective disorders, and quality of life? Heart. 2001;85(4):375-379.

17. Lelakowski J, Podolec P, Majewski J, Szczepkowski J, Olszowska M, Przewlocki T, Syty M, et al. [Quality of life in patients after implantation of pacemaker type DDD]. Pol Merkur Lekarski. 2000;9(50):554-556.

18. Figueroa C, Alcocer L, Ramos B. Psychological intervention to modify anxiety, depression and quality of life in patients with an implantable pacemaker. Psychology. 2016;7:374-381.

19. Norgaard ML, Melchior T, Wagner T, Haugan K. Suicide attempt by complete self-removal of a 12-year-old permanent pacemaker system: case report. J Cardiovasc Electrophysiol. 2014;25(1):99-100.

20. Bordier P, Robert F. Suicide by self-removal of a pacemaker. Am J Forensic Med Pathol. 2004;25(1):78-79.

21. Aquilina O. A brief history of cardiac pacing. Images Paediatr Cardiol. 2006;8(2):17-81. 